\title{
Contractive tension force stack actuator based on soft dielectric EAP
}

\author{
Gabor Kovacs ${ }^{a *}$, Lukas Düring a \\ ${ }^{a}$ Laboratory for Mechanical Systems Engineering, Swiss Federal Laboratories for Materials Testing \\ and Research (Empa), Ueberlandstrasse 129, CH-8600 Dubendorf
}

\begin{abstract}
Among the electronic polymers EAPs especially the dielectric elastomers are functional materials that have promising potential as muscle-like actuators due to their inherent compliancy and good overall performance. The combination of huge active deformations, high energy densities, good efficiencies and fast response is unique to dielectric elastomers. Furthermore, they are lightweight, have a simple structure and can be easily tailored to various applications.

Up to now most scientific research work has been focused on the planar expanding actuation mode due to the fact that the commercially available acrylic material VHB $4910(3 \mathrm{M})$ can easily be processed to planar actuators and has demonstrated very high actuation performance when pre-strained. Many different actuator designs have been developed and tested which expands in plane when voltage is applied and shrinks back as soon as the applied charges are removed from the electrodes.

Obviously the contractive operation mode at activation is required for a wide range of application. Due to the principle of operation of soft DE EAP, mainly two directions to performed work against external loads are possible. Beside of the commonly used expanding actuation in planar direction the contractile actuation in thickness direction of the DE film represents a very promising option in the multilayer configuration. First approaches have been presented by the folded actuator design and by the multilayer tactile display device.

In this study a novel approach for active structures driven by soft dielectric EAP is presented, which can perform contractive displacements at external tensile load. The device is composed of an array of equal segments, where the dielectric films are arranged in a pile-up configuration. In order to maintain satisfying structural integrity when external tension load is applied special attention was paid to the compliant electrode design which takes a central importance concerning the force transmission capability between each layer of the actuator. Due to the stack configuration of the actuator the commonly used and pre-strained acrylic film was replaced by the stress-free IPN modified acrylic film in order to eliminate the need for external pre-strain-supporting structures.

Introductorily, the specific problems on conventional expanding actuators are discussed and the aims for contractive tension force actuators are specified. Then some structural design parameters are addressed in order to achieve a high rate of yield and reliable working principle. In the main part of the study the manufacturing process of the actuators and some measurement results and experiences are discussed in detail.
\end{abstract}

Keywords: Adaptive structures; contractive tension force stack actuators; electro_active polymers; soft dielectric EAP; dielectric elastomer

\section{INTRODUCTION}

Among the different classes of active materials, especially the soft dielectric EAP, a subgroup of the electroactive polymers (EAP), are promising for muscle-like actuators ${ }^{1,2,3,4}$ due to their inherent compliancy and good overall performance. The combination of large active deformation (strain up to $380 \%$ in area), high energy density $\left(3.4 \mathrm{~J} \mathrm{~g}^{-1}\right)$ and prompt response is unique to dielectric elastomer (DE) actuators.

Soft dielectric EAP correspond to a compliant capacitor, where a dielectric elastomer film is sandwiched between two compliant electrodes (Fig. 1). When an electrical voltage is applied between the electrodes, the electrostatic forces (Maxwell stresses) from the charges on the electrodes squeeze the elastomer in thickness direction. Thus, the incompressible film expands in the plane. For this reason all actuators based on this working principle are denoted as

Electroactive Polymer Actuators and Devices (EAPAD) 2009, edited by Yoseph Bar-Cohen, Thomas Wallmersperger, Proc. of SPIE Vol. 7287, 72870A · C 2009 SPIE · CCC code: 0277-786X/09/\$18 · doi: 10.1117/12.815195 
expanding actuators. According to Pelrine et al. ${ }^{5}$ the equivalent pressure $\mathrm{p}_{\mathrm{eq}}$ of the electrodes grows linearly with the dielectric constant of the film and in square with the applied electrical field (eq. 1). As soon as the voltage is switched off and the electrodes are short-circuited the film deforms back to its initial state.

$$
P_{e q}=\varepsilon_{0} \cdot \varepsilon_{r} \cdot\left(\frac{U}{d}\right)^{2}
$$

Thereby $\varepsilon_{0}$ is the free-space dielectric permittivity $\left(\varepsilon_{0}=8.85^{*} 10^{-12} \mathrm{~F} / \mathrm{m}\right), \varepsilon_{\mathrm{r}}$ is the relative permittivity of the material and $\mathrm{d}$ represents the thickness of the DE film.

$$
\begin{gathered}
\text { Deactivated } \\
(U=0)
\end{gathered}
$$

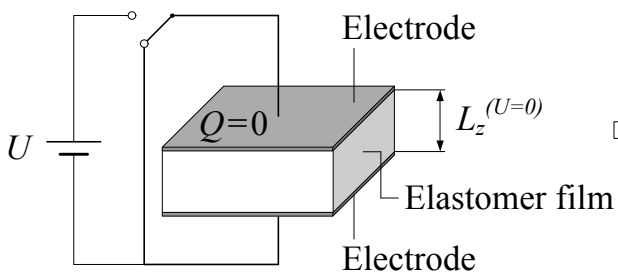

Activated

$(U>0)$

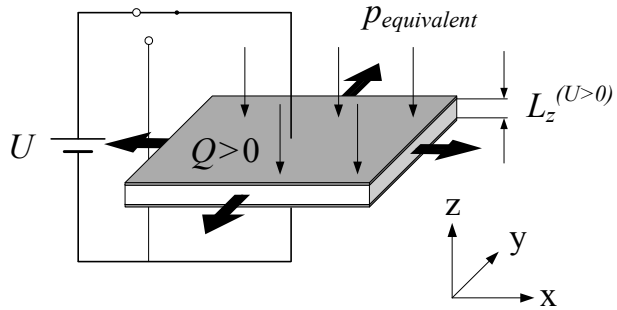

Fig. 1 Structure and principle of operation of soft dielectric EAP

This outstanding performance has been achieved with the acrylic films subjected to large biaxial pre-strain that enhances the polymer's specific dielectric breakdown strength for a given thickness of the material ${ }^{6,7,8,9,10,11}$. Due to the hyperelastic trend of the acrylic's Young's modulus a unidirectional activation with maximal performance can be achieved by considering a non-uniform biaxial pre-stretching. Based on this fact the pre-stretch ratio $\lambda$ represents one of the most relevant parameters to maximize the axial actuator elongation. High unidirectional performance activation for most actuator types can be achieved when the two pre-stretching ratios $\lambda_{\mathrm{x}}$ and $\lambda_{\mathrm{y}}$ are optimized ${ }^{17}$. As a result this pre-strain of the acrylic film has to be maintained on any actuator and a rigid supporting structure is required where the acrylic film is circumferentially fixed. For enabling the required displacements with soft dielectric EAP, this support structure must offer the corresponding mechanical degrees of freedom (DOF). Obviously, with pre-strained DE actuators the design of the support structure is one of the key issues and represents many design constraints. Nevertheless a variety of different types of dielectric elastomer (DE) actuators demonstrated the versatile capabilities of this actuator technology (e.g.12,16). Due to their intrinsic compliance and unique deformation potential, soft dielectric EAP are promising for compliant, lightweight structures in the macro-scale, which can perform continuous displacements.

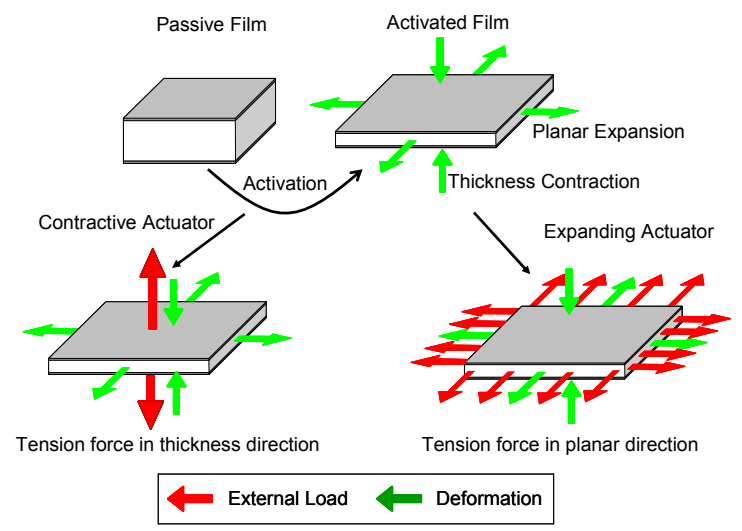

Fig. 2 Actuation modes: In-plane expanding - out-of plane contractive actuation 
When high actuation force is required up-scaling of the actuators' forces becomes inevitable. This can be accomplished by arranging many dielectric films in parallel. One simple way to reach such stacking of films is to roll a pair of DE film around an elastic core. This very promising actuator configuration is denoted as (spring) roll actuator and exhibits an uniaxial elongation when activated ${ }^{12,13,14,15}$. Presently reported elongation potential of spring roll actuators is in the range of $34 \%$ and forces of up to $15 \mathrm{~N}$ were achieved. In connection with the general actuation modes of the dielectric elastomer film they can be used in different ways. Referring to the principle of operation of soft dielectric elastomer actuator (Fig. 1), mainly two directions to perform work against external loads are possible:

- Work in planar directions (expanding actuator): Under electrical activation of a DE basic unit the film expands in planar directions and can thus work against external pressure loads in both planar directions.

- Work in thickness direction (contractile actuator): Under electrical activation the electrodes squeeze the DE film in thickness direction. Thus, the actuator can work against external tensile loads acting in thickness direction.

So far most scientific research work has been focused on the planar expanding actuation mode due to the fact that the commercially available acrylic material VHB 4910 (3M) can easily be processed to planar actuators and has demonstrated very high actuation performance when pre-strained ${ }^{5,7}$. Many different actuator designs have been carried out for experimental purposes and represents the present state-of-the-art, as they are the extender (planar), unimorph-, bimorph-, spring roll-, push-pull-, bow-tie-, diamond-, diaphragm-, spider-, inchworm segment- and universal muscle actuator. They all have the same basic electro-mechanical characteristic: The actuator expands in plane when voltage is applied and shrinks back as soon as the applied charges are removed from the electrodes. The development of the arm wrestling robot in 2005 has produced a major contribution to the production technology of expanding actuators in rolled configuration. For the arm wrestling competition (AWC) at the SPIE conference in San Diego 2005 the Empa made arm robot has demonstrated the capability of the EAP actuator to be used in a system which can produce remarkable mechanical work ${ }^{17}$. Due to the expanding actuation characteristic an agonist- antagonistic arrangement of the actuators was necessary to produce a bidirectional motion. In this configuration all actuators were fixed in a pre-strained state in order to execute the required contracting motion under external tensile force in the deactivated mode. Without exception all highly pre-strained actuators with high actuation performance in planar direction (force and elongation) have poor long-term lifetime due to the permanent high pre-stretching in combination with the necessary rigid, heavy and bulky supporting structure.

When contraction at activation is required the nowadays well known "expanding planar EAP actuator" does not represent the appropriate solution for many applications. The basic DE working principle can be exploited in order to build linear contractile actuators, according to a few device configurations. A few approaches have been carried out to provide actuators with contractive motion property when activated and are briefly explained as follows:

Based on the folded and helical design with non-pre-strained silicone film contractive motion of the actuator has been demonstrated ${ }^{18}$. The so-called folded actuator consists of a single strip of an elastomer that is first coated with compliant electrodes and then folded up, so as to form a monolithic compact body. The strip is successively coated with continuous compliant electrodes, made of a silicone/carbon-black mixture. In the case of the silicone elastomer, the folded structure is finally 'sealed' with a thin coating made of the same elastomer used for the strip. A high voltage applied between the electrodes causes a thickness squeezing of the entire elastomeric layer which results in an axial contraction of the overall structure, along with related lateral expansions. Furthermore the basic design of the stack configuration has been developed and demonstrated for electrostatic tactile displays with high structural compliance ${ }^{19}$. When a voltage is applied between neighbouring electrode layers of this device, the actuator stack will contract and the stimulator tip will disappear below the surface of the device. Due to the fact that the finger tip is basically pressing on the tip no essential tension force has to be produced by the actuator structure for the pull-back motion. By reducing the voltage the electrodes are discharged beyond the voltage source causing a relaxation of the actuator stack. Thereby the stimulator tip is pressed against the skin on top of the device because of the stored elastic energy.

In contrast to this actuator design, a new actuator in pile-up design is proposed which exhibits contractile deformation under electrical activation and additionally can work against external tensile loads acting in contractive direction. The actuator design and working principle is also based on the contraction effect in thickness direction of the dielectric film when an electrostatic field is applied. Since the actuation direction is parallel to the electrostatic field lines the electrostatic force can directly be turned into mechanical force. The as compressed DE film produces the contraction motion of the entire actuator. To provide the tensile force transmission from one DE layer to the next the electrode design plays a central role and represents the promising core technology in the field of soft dielectric EAP actuators. In 
contrast to the expanding planar actuator in different configurations, the proposed contractile actuator demonstrates the feasibility and convenience of the pile-up configuration for many applications where contraction when activated and production of tensile force is of central interest.

\section{CONCEPTUAL APPROACH}

The proposed contractive actuator consists of stacked small DE films pieces coated with compliant electrodes. The electrostatic field induced actuation force and displacement is normal to the plane of the electrode and dielectric film. The as obtained contraction of the actuator can be used to drive the device under a specific service load. The design of the pile-up actuator is based on a series of capacitors electrically connected in parallel with alternating polarities of each layer. When transmission of the external tension force is required the internal force (stress) distribution of the actuator is of central interest. Thereby the actuator has to be capable to transmit the external tension force from one load introduction point, placed at the end of the actuator, to the second one positioned at the opposite end. This requirement is of paramount importance when the actuator is activated and contraction motion is supposed to be executed under external tension force. In normal case tension stress state occurs for most structures consisting of solid material when loaded. Due to the composed structure of the proposed actuator consisting of a series of dielectric elastomer film and compliant electrode in alternating order the general situation is different:

When a voltage is applied between two electrodes external tension force can be transmitted by the electrostatic field induced Maxwell force. As a result the dielectric elastomer is squeezed by both neighboring electrodes and contractive motion of the entire actuator occurs. Although considerable external tension load can be transmitted by the electrostatic force solely compression stress is triggered within the dielectric film. In this configuration the dielectric film acts as a "spacer" and does not take any tensile stress (neither in planar nor in normal direction). As a beneficial property this issue is an important aspect when good fatigue strength under cyclic tension force loading and long term stability of the actuator is of central interest. As it is well known, the electro static field vanishes inside the electrode due to its desired high electric conductivity. In order to provide even so the tension force transmission within the electrode the electromechanical properties of the electrode is of central interest and is discussed as next from a constitutive point of view.

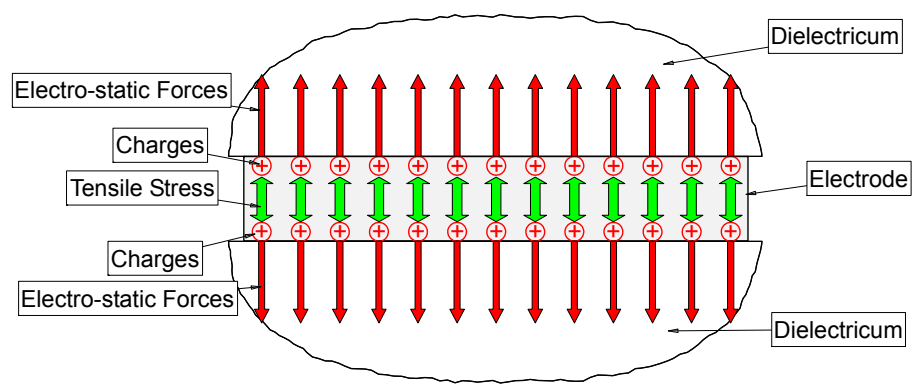

Fig. 3 Mechanical tensile stress within the electrode

Assuming absent electrostatic field the external tension force has to be transmitted over the electrode layer by producing tension stress inside the electrode material (as sketched in Fig. 3 by the green arrows). This can be easily accomplished when compressible solid material is considered for dielectric medium. In such a case no planar expansion occurs when compressed by the Maxwell force and therefore the electrode can be made of solid conductive material (stiff plate with high conductivity). When incompressible material like hyper-elastic elastomer film as dielectric is applied, the electrode has to be compliant in planar direction in order to allow unrestricted planar expansion of the dielectric film. In this case the conductivity of the electrode has to be maintained without reducing the electrostatic field even in the deformed state. 
On the other hand and in contrast to conventional dielectric elastomer actuators, the electrode has to be designed in such a way that the tension force transmission capability in normal direction of the film is assured. For this reason stiff deformation property of the electrode material in transversal direction (parallel to the electrostatic field) is required to perform mechanical tension stress. In order to fulfill both electro-mechanical requirements of the electrode, anisotropic material with direction dependent stiffness has to be applied. The following explanation of the working principle is based on an idealized and macroscopic layered system with homogeneous properties. When voltage is applied between all electrodes the charges are arranged on the surface of the electrode material and thus located nearby of the dielectric film. The resulting attracting forces of the opposite pole charges are producing tension force between both surfaces of the oppositely positioned electrodes. Accordingly the same attracting forces are creating a mechanical tension stress inside all electrode layers (Fig. 3). By this means the electrostatic force can be transmitted from one side to the opposite side of the electrode by the resulting tension stress state of the electrode material, which needs a high stiffness in transversal direction. Any adhesive bonding effects between the dielectric and electrode layers might be helpful for mechanical stability in passive state but not necessary for the force transmission at activation. Consequently an anisotropic material system has to be used as electrode with direction dependent stiffness in order to fulfill the in-plane compliancy and the out-of-plane stiffness and strength demand.

\section{THE STACK ACTUATOR}

\subsection{The actuator design}

The proposed actuator is based on a series of elastic capacitors consisting of many small pieces of dielectric elastomer films coated with compliant electrically conductive material as electrode. They are layered in serial configuration but electrically connected in parallel with alternating polarities of each layer (Fig. 4). Thereto each electrode has an appendix to the border of the corresponding layer. Alternating polarities can be achieved by arranging the electrodes in alternating alignment mode of the appendices when piled up. The appendices are connected with a compliant conductive band placed on two opposite side faces of the actuator addressing the associated conductive layers. This electrode attachment configuration depicts an essential impact on the low electric resistance of the entire system. For activation the main feeding (supply) line of the voltage source has to be attached to the actuator, electrically connecting each electrode layer. In this configuration, where the actuation direction is parallel to the electrostatic field lines and therefore normal to the plane of the electrode and dielectric film, the electrostatic force can directly be turned into specific mechanical service force to drive the device. Due to the pile-up design of the actuator no external supporting structure is possible in order to maintain the stress of each film in a useful way. Hence DE film material has to be applied, which shows good electromechanical performance in stress free state.
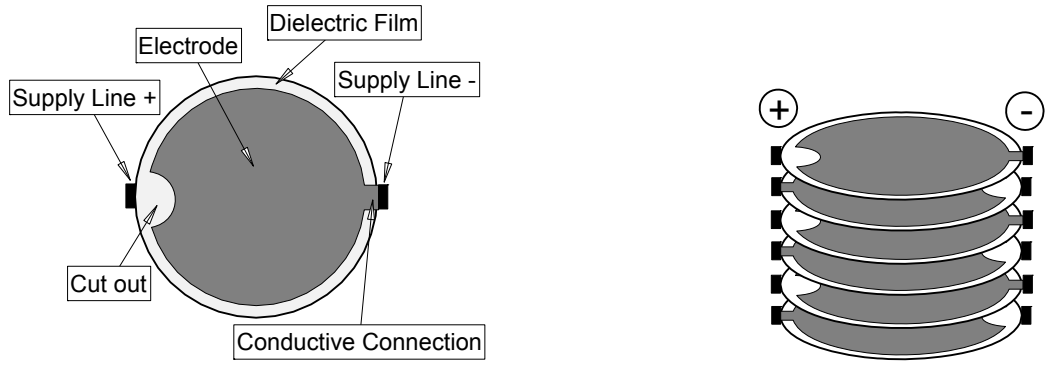

Fig. 4 Stacked configuration of dielectric elastomer layers

Due to reliable operation of the device, the shape of the applied electrodes is of central importance and represents one of the major design parameter. In this context a small passive zone as a circular cut out section has to be taken into account 
near to the brink of each layer in order to ensure the electric separation of each electrode. Furthermore the pull-in effect (with high risk of electric breakdown) has to be avoided by alternating offset of the electrode edges due to the electrostatic field concentration density at the edges of each electrode. The insulation capability can be increased by closing the gap (passive area) between the two dielectric elastomer layers. This can be accomplished by mechanically compressing the whole actuator in axial direction during the manufacturing process. Thereby it is of central importance that the electrode is essentially thinner than the dielectric film (at least two magnitudes thinner).

Beside of the electro-mechanical properties of the dielectric film and the electrode material the performance of the actuator is essentially determined by the following 3 main design parameters:

- The number of applied layers affects the length of the actuator and therefore the absolute stroke when activated. Based on the produced electrostatic pressure the absolute exhibited force is determined by the area of one layer of dielectric elastomer film. By adding a large number of equal elastomer and electrode layers a macro scale actuator can be produced.

- The envisaged service force has to be introduced into both ends of the actuator which requires appropriate end fixing parts consisting of stiff material. The interface between the soft actuator material and the stiff end fixing part leads to a locally multi-axial stress state of the DE material. As a consequence the actuator performance is reduced within this transition zone with limited deformation capability.

- To avoid electric breakdown at the side surface between the electrodes, an uncoated boarder area is necessary (Fig. 4). As a result a loss of electro active area has to be considered, which generally reduces the effectiveness of the actuator. Therefore the width of the passive boarder has to be designed as small as possible in order to obtain maximum performance.

The passive, uncoated material area generates a mechanical resistance due to its elastic property and is a contra productive effect respecting the actuation performance. Additionally a clearance (cut out) of the coated electrode has to be considered where the supply line with opposite polarity is placed to avoid any electric breakdown. For this reason the ratio of the uncoated and coated area essentially determines the effectiveness of the actuation. As a matter of fact it is of central interest to decrease the size of the passive area to a minimum required width. However, the required minimum width of the passive area is determined by the applied voltage amplitude and the electric breakdown strength of the dielectric film. Consequently the size of the passive stripe represents an absolute value and does not depend on the size of the actuator's cross-sectional area. Referring to the ratio of the uncoated and coated area, actuators with large crosssectional area are preferred accordingly.

This effective actuator configuration represents a fault tolerant system because every eventually broken layer can be localized and switched off the electric system. Tests on failed devices have shown that malfunctioning actuators can be repaired by replacing or just removing single broken layers. This can be achieved by easily dismounting the actuator close to the place of the failed layer and re-assembling it again after having fulfilled the repair.

\subsection{The manufacturing process}

As basic material single dielectric elastomer film discs are used for the pile up process, which are coated on one side. The challenge in terms of processing and handling lies in the practical implementation of the dielectric material and the realization of their theoretical potential (up to $40 \%$ contraction and $0.16 \mathrm{~N} / \mathrm{mm} 2$ actuation pressure). With regard to the actuator configuration (high number of layers) efficient manufacturing process of the material system at high precision becomes to a key importance. Furthermore special attention has to be paid to the design of the load introduction parts at both ends of the actuator (determination of the film shear stress deformation by modeling the hyper-elastic DE film).

Due to the pile-up design of many small DE layers no pre-strained film equipped with the necessary strain supporting structure can be processed in a useful manner. However, the pre-stretching stress has to be eliminated to obtain a stack actuator with acceptable specific performance. This has been achieved by applying the interpenetrating polymer network (IPN) post-process, which can effectively support the pre-strain of the acrylic film. Due to the very promising property most EAP stack actuator were made with IPN post-processed VHB 4910 films $^{20}$. As electrode material pure Ketjenblack KJ-600 graphite powder has been established for all types of EAP actuators made by adhesive films. The powder is smeared on the sticky surface of the film. The as obtained thickness of the applied coating is in the range of 50nm ${ }^{21}$. 
According to the IPN process the film was stretched to 5 times its original length and width, or $400 \%$ biaxial strain. The pre-stretching has been accomplished on the biaxial pre-stretching machine established at the Empa laboratory. After the film stretching process a film supporting frame was fixed to the stretched film and subsequently cut out and removed from the pre-stretching machine. The TMPTMA monomer was dissolved in ethyl acetate and than sprayed onto both biaxially pre-strained VHB films still fixed in the frames. After two days of rest period for penetration the films were than placed in a vacuum oven and heated up to $85^{\circ} \mathrm{C}$ to induce free-radical polymerization thereto. A large volume vacuum chamber equipped with thermal radiation plates was used for the curing process. After 6 hours being cured in the vacuum oven the film is still fixed in the supporting frame and therefore under tension stress. In case of automated manufacturing method the film was removed from the frame and relaxed in order to obtain a stress free material. For the handcrafted manufacturing process the film was fixed on a flat Teflon supporting plate. In this state the film represents an ideal condition to manually apply the first coating layer.

In general many small and coated DE film pieces have to be assembled in a stack configuration. The quality of the stack actuator in terms of actuation performance essentially depends on the appropriate pile-up manufacturing process. Thereby high accuracy of the coating, cutting and pile-up process is of paramount importance to avoid any local breakdown effects when in use. In order to follow theses requirements two different favorable methods have been evaluated and established to produce stack actuators and are explained as follows:

\section{Parallel processing (Handcrafted)}

As long as no automated manufacturing facility is established handcrafted actuators is the only one option. Obviously a sophisticated and efficient processing technique is required to produces a stacked actuator consisting of thousands of layers, depending on the envisaged entire length. In order to assure the required high precision within a reasonable time parallel processing in matrix architecture has been turned out as the appropriate procedure. Thereby modules consisting of approx. 50 layers are produced representing the most efficient method. Consequently large units of DE film were used for coating many small active zones at once (Fig. 5, left).
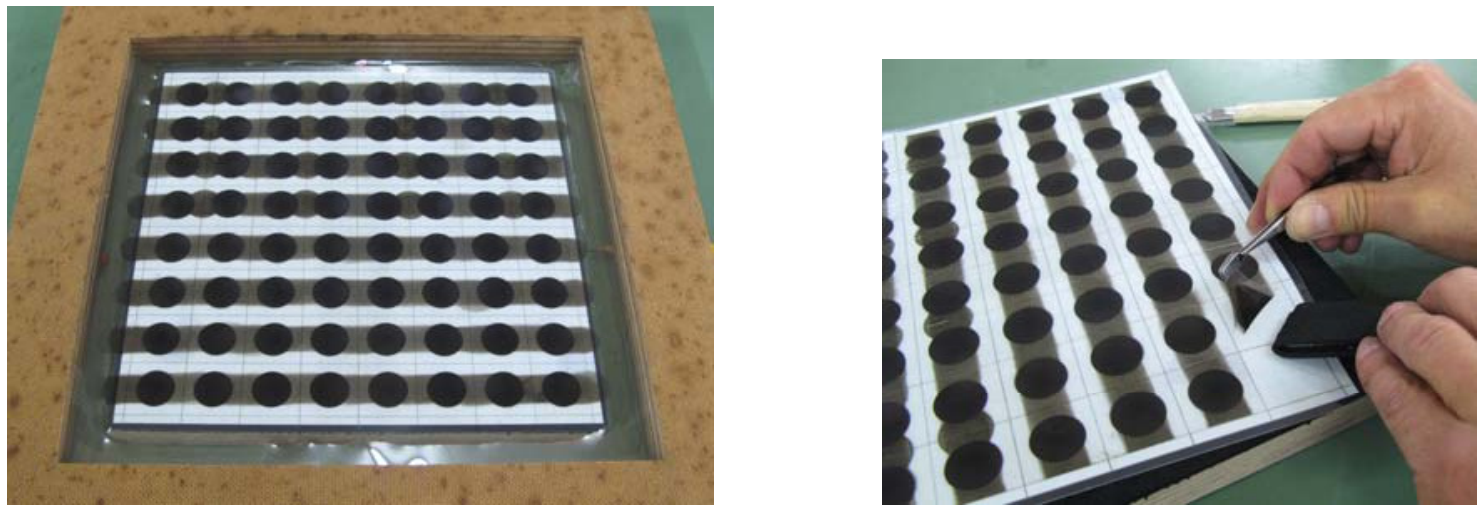

Fig. 5 Parallel processing in matrix configuration (left), removing each sample for the pile up process (right)

By stacking a low number of these films (in the range of 10 pieces) many actuator layers can be produced in a few preliminary process steps. Thereby each film is coated with electrode material by using a mask which defines the shape, size, location and finally the number of pieces placed on this sheet, representing respective actuator packages. By cutting out each package, removing from the supporting plate (Fig. 5, right and Fig. 6, left) and piling them up the final modules can be accomplished (Fig. 6, centre). 


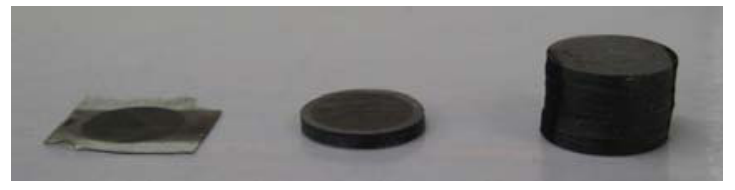

Fig. 6 Intermediate steps of the handcrafted manufacturing method with parallel processing

This manufacturing method in parallel process mode has been evaluated as the appropriate handcrafted manufacturing method. These pre-fabricated modules can easily be assembled to an entire actuator (Fig. 6, right). Accordingly the length of the actuator can be adjusted by stacking the appropriate amount of modules.

\section{Batch processing (automated facility)}

For most manufacturing activities on industrial level, however, a fully automated process is strongly preferred. For this reason a prototype machine was developed and set up to produce stack actuators in a mostly automated mode (Fig. 7). To demonstrate the feasibility of an automated DE actuator fabrication the present "stacker" machine has been developed at Empa. Thereby the actuator is produced by sequentially stacking, cutting and coating DE films layer by layer. Using this method the actuator is "growing" to the required size (length) at given cross sectional size (max. 50mm in diameter) and shape. In contrast to the handcraft method only fully relaxed dielectric film can be used as feed material.
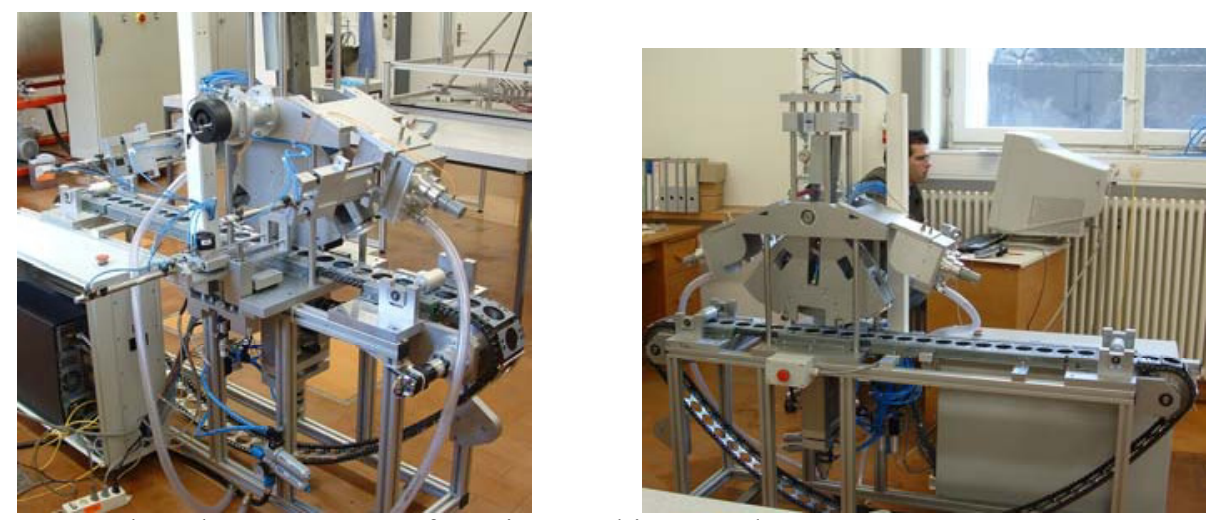

Fig. 7 Fully automated stack actuator manufacturing machine "stacker"

Due to practical reasons modules are produced as well with limited number of layers $(<200)$. Unlike to the handcrafted method high repeatability and constant manufacturing quality can be provided by using the stacker machine with repeating process cycles. The assembly of the modules and the finish of the actuator represent the final steps of the process. After the stacking process the actuator has to be equipped with two adapter fittings at both ends where the longitudinal external force can be applied. Due to the fact that the acrylic film is an adhesive material (specification of $3 \mathrm{M})$ we took advantage of the sticky property of the film.

For both methods carbon powder Katjenblack EC-600JD was applied to the film by a smearing brush. As mentioned before it is an essential aspect to produce an ultra thin conductive layer in terms of anisotropic property. For this reason the adhesive property of the acrylic film was utilized to affix the minimum amount of powder on the surface of the film. The surplus amount of powder could be removed by a vacuum cleaner. The as obtained compliant conductive layer has a thickness of a few hundred nanometers and therefore semi transparent.

The connecting strips on the side faces to electrically contact the electrodes are applied by the same method as the electrodes themselves. For big actuators with high capacity the conductivity of the connecting strips is not good enough for fast charging/discharging and therefore not appropriate for high performance applications. This can be improved by 
placing additional soft copper wires on the carbon powder areas. These wires were fixed with rubber bands wrapped around the actuator and as a result a good electrical contact was assured.

The adhesive property of the acrylic was used to fix a non-conductive screw head onto both end faces of the actuator for the foreseen external tension force introduction. This proposed layout represents a very compact, robust and easy to handle system.

The as obtained actuator is now ready for service. The actuation voltage can be applied by two metal bands positioned on both connecting strips (Fig. 8) or when installed by connecting the copper wires. The as obtained actuator is now ready for service.
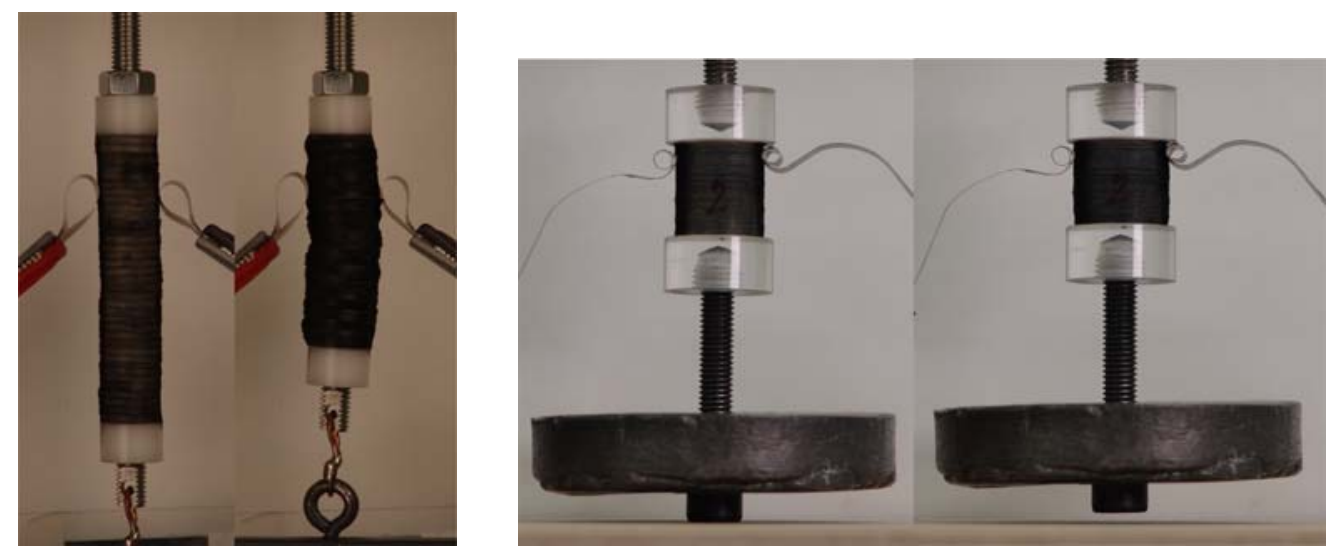

Fig. 8 Two stack actuators in action: left half in passive mode - right half in activated mode.

\subsection{Experimental characterization}

The performance of the actuator was experimentally determined in terms of the static contraction performance when activated under different condition, as they are

- Free contraction of the entire actuator (no external constrains and loads)

- Unloaded contraction of the actuator equipped with fasteners at both terminal points

- Unloaded contraction of the entire actuator consisting of different passive/active area ratios

- Contraction of the actuator under different external tension forces (accordingly equipped with fasteners at both terminal points)

In order to demonstrate the design parameter dependency on the actuation performance and the deformation potential respectively two actuators with circular punched layers at different electrode design and size are presented. The key specifications of the actuators are described in the following table 1.

\begin{tabular}{|l|l|l|l|l|}
\hline Actuator & Total diameter & Active diameter & Height & Number of layers \\
\hline 1 & $18 \mathrm{~mm}$ & $16 \mathrm{~mm}$ & $18.3 \mathrm{~mm}$ & Approx. 280 \\
\hline 2 & $20 \mathrm{~mm}$ & $16 \mathrm{~mm}$ & $21.2 \mathrm{~mm}$ & Approx.330 \\
\hline
\end{tabular}

Tab. 1. Key data of both characterized actuators.

To determine the influence of the stiffening effect at both terminal points each actuator was once actuated without fasteners (Fig. 10) and once actuated with fasteners (Fig. 11). As additionally design parameter actuator \#1 was 
manufactured with a passive boarder width of $1 \mathrm{~mm}$, which has reviled the difficulties of high precision processing. All other actuators were produced with $2 \mathrm{~mm}$ width of uncoated area.

Obviously both actuators depict an essential contraction offset when actuated at different edge condition. For both tested actuators the stiffening effect of the fittings positioned at both terminal points is not negligible. However it has to be pointed out that all actuators were relatively short in comparison to the diameter. In this case the transition zone between the stiff part and the freely deformable region is relatively large compared to the entire length of the actuator. This effect can be visually observed on the actuator depicted in Fig. 11, which is very different to the "slim" actuator in Fig. 8 left, where the length to width ratio is essentially larger. As expected the curves in Fig. 9 reveal the performance dependency of the passive/active area ratio as the most relevant design parameter. The maximal measured contraction for free end actuators was $46 \%$ at an activation voltage of $4.1 \mathrm{kV}$. This was achieved with actuator $\# 1$ which has the smallest amount of passive area (21\%). Actuator \#2 has reached a lower contraction up to $35 \%$ at even higher activation voltage of $4.2 \mathrm{kV}$.

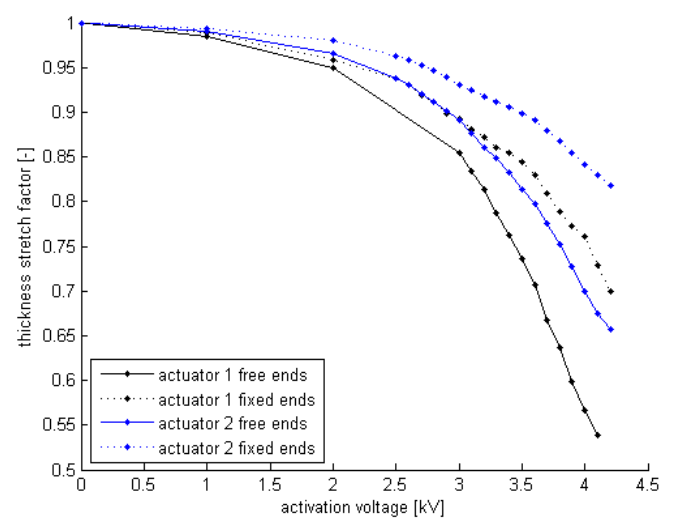

Fig. 9 Comparison of the measurement data for free end actuators and unloaded fixed end actuators

The same test was carried out where the actuators were equipped with fittings. The maximal measured contraction for actuator \#1 was $30 \%$ at an activation voltage of $4.2 \mathrm{kV}$ whereas actuator $\# 2$ reached a contraction up to $20 \%$ at the same activation voltage. Due to missing fasteners at both terminal points the deformation of the actuator can take place without any external constrains. But, the passive (none coated) circular boarder stripe does not participate at the contraction motion and acts as a deformation resistance. However, the inner active part contracts when activated and simultaneously expands in planar direction. As a result the surface of the actuators face side displays a circular bathtub like shape, which is well observable in Fig. 10. In this configuration the actuator has shown considerable high contractive deformation, which can never be achieved with mounted fasteners.
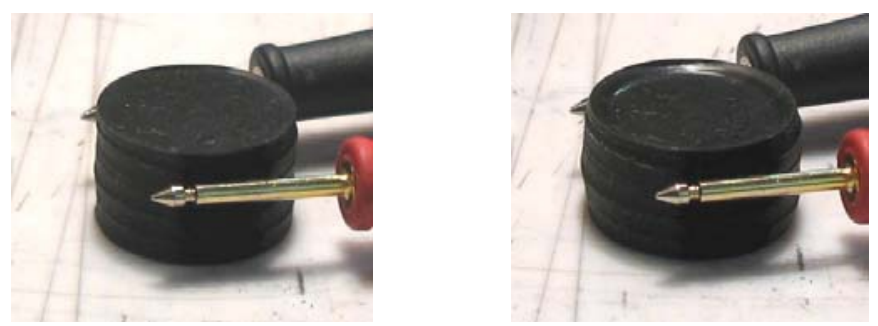

Fig. 10 DE stack actuator with free end: deactivated (left) - activated (right)

The performance impact of passive boarder size as well as the stiff end fixing parts is remarkable and therefore represents the major design criteria. Assuming that most actuators in praxis will be loaded with external forces the actuators have to be equipped with appropriate fittings, operating as load introduction parts. For this reason the significant difference in maximal contraction between free end and fixed end actuators has to be taken into account for the designing process. This conclusion for sure is true for actuators with small form factors (length to width ratio). On 
the other hand this stiffening effect is negligible for long and slim shaped actuators. For a DE stack actuator with a larger length to width ratio and a smaller amount of passive border area to total cross sectional area a smaller difference is expected.
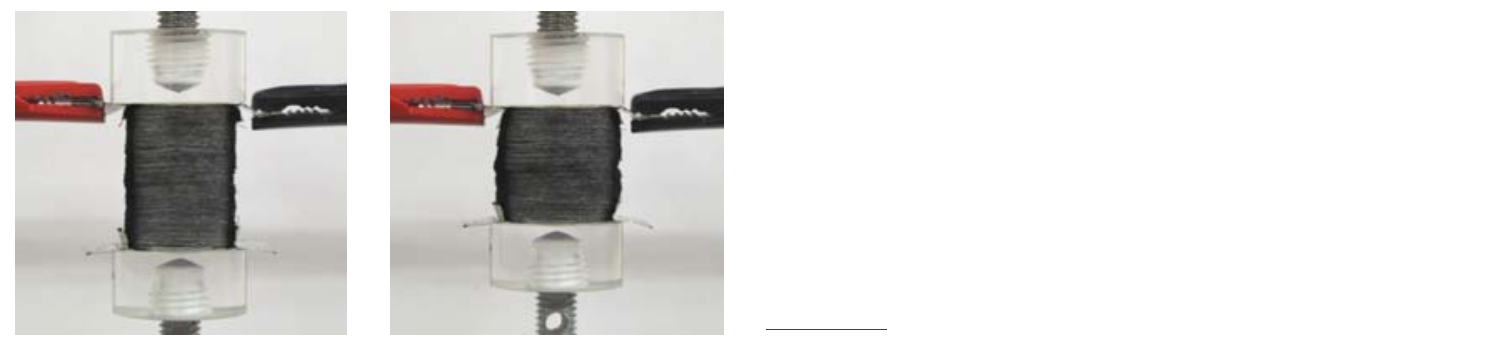

Fig. 11 DE stack actuator equipped with fixed end parts: deactivated (left) - activated (right)

With regard to any potential application the contractive deformation of the actuator under different external tension loads is of central interest. For this reason we have carried out some tensile activation tests with actuator $\# 5$ in order to gain first findings about the electro-mechanical property of the contractive stack actuator abandoned to external tensile force. As a matter of course the tested actuator was provided with appropriate fasteners at both terminal points for the tension force introduction (Fig. 11). In the vertical position the upper end of the actuator was fixed to a stiff frame whereas the load, in this case a weight, has been attached to the lower end of the actuator. It is important to emphasize that the actuator was not loaded in the passive state before starting the actuation tests. Accordingly the weight was positioned on the top of a fixed supporting plate in the passive state. At activation the weight was lift off by the contraction motion of the actuator. Applying different voltages in static mode the particular vertical displacement of the weight was measured, representing the appropriate deformation of the actuator.
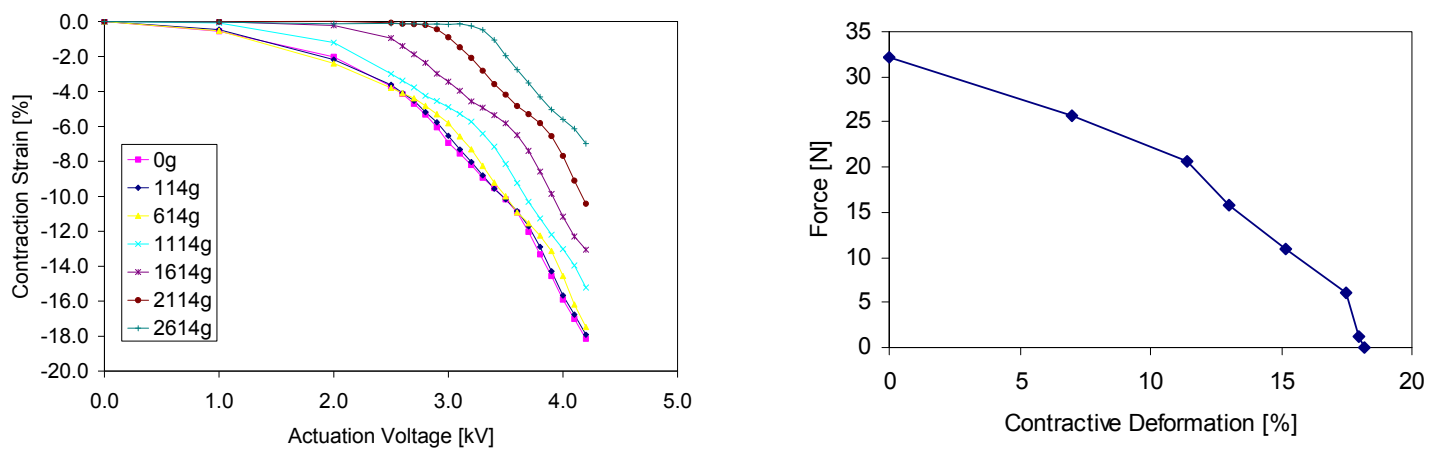

Fig. 12 Contraction of DE stack actuator at different actuation voltage and external load (left diagram). Connecting the end points of each curve the force-contraction curve is displayed (right diagram)

In the left diagram of Fig. 12 the typical "lift-off" effect can be observed, which is characterized by the delay of contraction motion at increasing voltage and given external weight. This effect can be clearly noticed for the case with highest load (2614g): The curve forms a sharp bend at $3.2 \mathrm{kV}$ actuation voltage. At this point the appropriate electrostatic field is created to produce the minimum "lift off" force in order to lift the weight. With further increasing of the voltage the contractive motion continues in similar manner than all other curves. Connecting all end points of the set of curves the most important force - deformation curve can be defined (Fig. 12 right), which is mostly relevant for the design process drawn to a specific application. Based on this characteristic, which is valid for a specific actuators shape and material, the whole area below the curve represents the field of possible applied actuation. 


\subsection{Design process}

The force - contraction curve (Fig. 13) comprises the most important information for engineering the actuator. This curve represents the upper limit of the applied external force, which is equivalent to the net actuation force. Obviously the maximum deformation is limited by the materials stiffness, where the Maxwell stress is in balance with the electrostatic force. In this situation no external force can be applied. On the other hand maximum force can be produced when no deformation occurs. Consequently all charge states are located within these two extreme deformation positions.

One proposal for the common approach of the designing procedure is following as next. As it is expected from the general behaviour of the dielectric elastomer actuation, Fig. 12 right depicts the typical non linear characteristic of the curve. In order to simplify the engineering process, this curve is defined in the conservative linearized form.

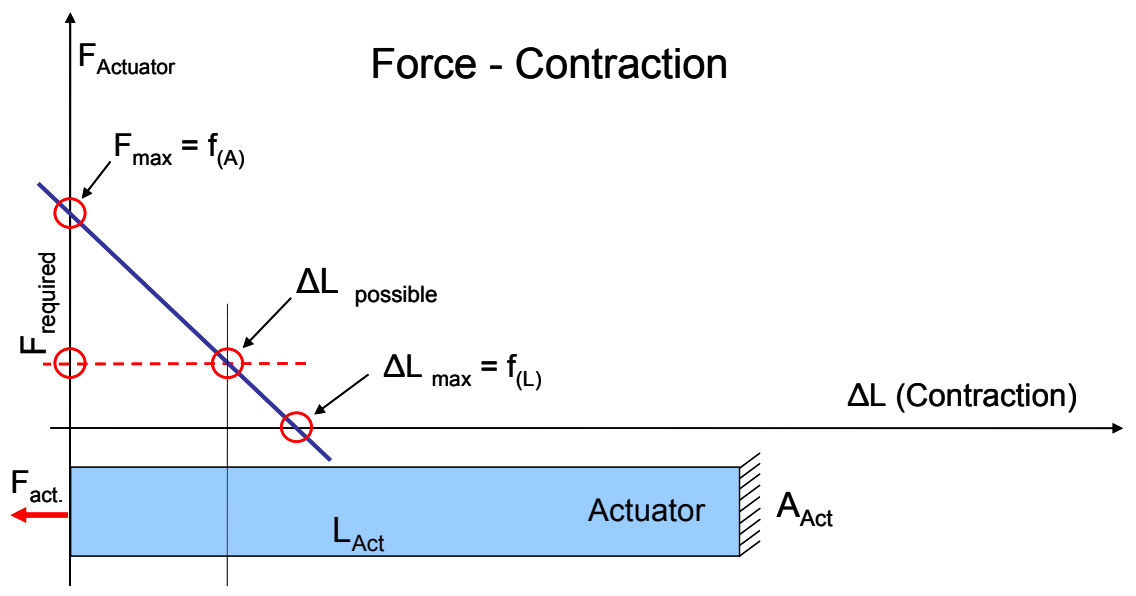

Fig. 13 Simplified force - displacement curve of a stacked actuator at applied external tension force

For a given length and cross-sectional area of a stack actuator the simplified contraction - force curve can be defined in the general form displayed in Fig. 13. All achievable work points are located below this curve. If higher values of the actuation force $\mathrm{F}_{\text {required }}$ at given contraction $\Delta \mathrm{L}_{\text {possible }}$ (or vice versa) is required two different measures are possible, as depicted in Fig. 14.
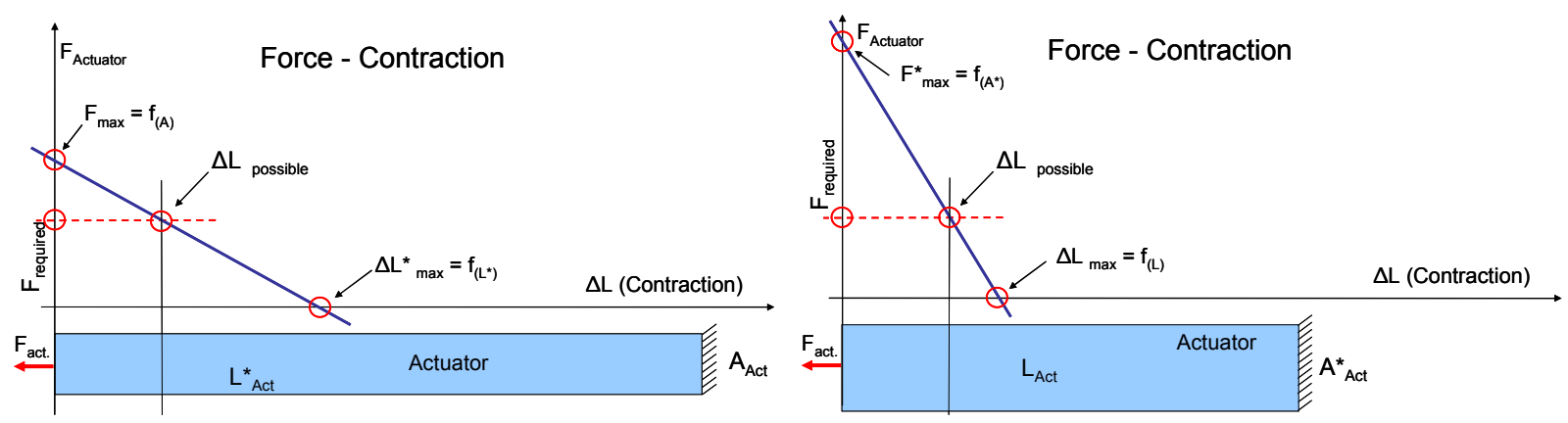

Fig. 14 Adapted force - displacement curves of a stacked actuator at a given external force $\mathrm{F}_{\text {required }}$. On left picture the characteristic curve has been truncated by using longer actuator for larger max. contraction $\Delta \mathrm{L}^{*}$. At given length of the actuator (right picture) the cross-sectional area can be increased in order to enhance the initial tension force $\mathrm{F}^{*}$. Both measures can be used in order to meet the required external force $F_{\text {required. }}$.

On the one hand the length $\mathrm{L}_{\mathrm{Act}}$ of the actuator can be enlarged in order to increase the absolute contraction length $\Delta \mathrm{L}^{*}{ }_{\max }$ (Fig. 14, left). Thereby the initial tension force $\mathrm{F}_{\max }$ at zero deformation is still the same, but the characteristic 
curve becomes less inclined. Thus the possible actuation force $\mathrm{F}$ in combination with the foreseen contraction $\Delta \mathrm{L}_{\text {possible }}$ becomes larger. On the other hand the initial force $\mathrm{F}_{\max }$ can be increased by enlarging the cross sectional area $\mathrm{A}_{\text {Act }}$ of the entire actuator (Fig. 14, right). By this means the length of the actuator does not have to be changed. As a result higher actuation force can be achieved as well on the basis of the steeper characteristic curve.

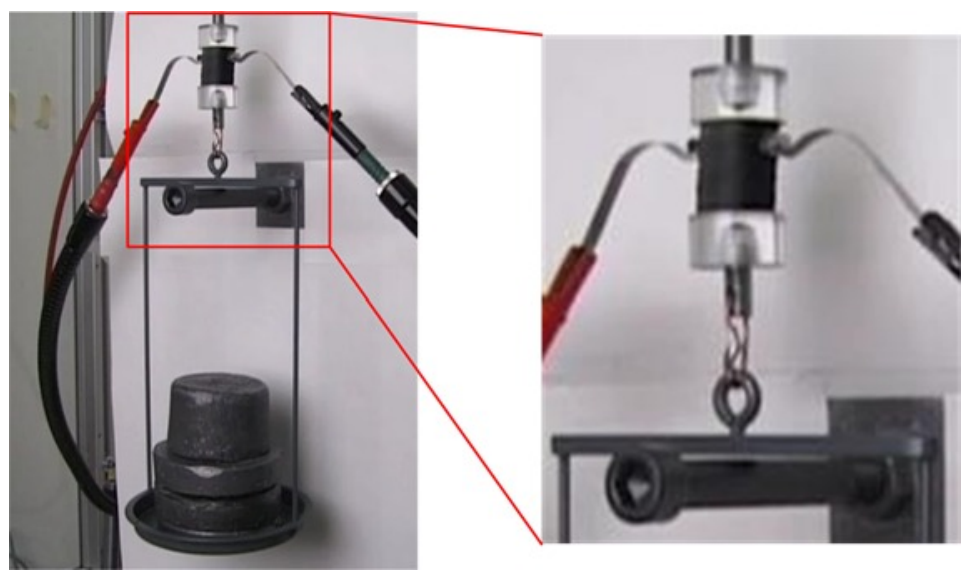

Fig. 15 Contraction of the DE stack actuator at $4.5 \mathrm{~kg}$ external load.

In order to demonstrate the potential of the proposed design the actuator was loaded with $4.5 \mathrm{~kg}$ mass to be lifted (Fig. 15). Approximately $5 \%$ of contraction has been observed which is close to the maximum possible actuation force.

\section{CONCLUSION}

A novel dielectric elastomer actuator design is presented with contraction capability under external tensile force when voltage is applied. Due to the pile-up configuration of stress free dielectric films and the anisotropic property of the applied electrodes the structural integrity can be controlled by the electro-static field induced Maxwell stresses. As presented a set of well working EAP stack actuators was successfully built and the working principle demonstrated. A first series of basic characterizations have shown considerable actuation performances of the stack actuator under attached external tensile force. Based on practical experiences the relevant design parameters have been evaluated and partly experimentally quantified. As most relevant finding the characterization showed that already a small change of length to width ratio and the amount of passive to total area results in significant performance change. Although external tensile force can be applied to the actuator each dielectric film is exposed to pressure stress due to the Maxwell forces when activated. This is beneficial when good fatigue strength under cyclic tension force loading and long term stability of the actuator is of central interest. After 1 year in operation and approx. 500 repetitive activations no failure due to fatigue can be reported up to now. A remarkable robustness against exterior influence, such as humidity and mechanical influences was noticed. Specific fatigue tests are planned in the near future.

Consequently, stacked dielectric elastomer actuators offer today the unique potential of enabling really innovative actuation systems where contraction at activation is of central interest. The specific properties of these devices make them particularly suitable for developing light, compliant and flexible systems at noise free actuation. This new type of actuator with high reliability represent the appropriate design when linear contractions of a soft actuator are required, in order to mimic the functional performances of human muscles, i.e. in applications where noise-free contractive actuation is needed and working in close distance to humans. In particular driving prosthetic limbs or robot arms and fingers as well as powering human machine interfaces is of immediate interest. 
It is straightforward to recognize that such applications can belong to very different disciplines, such as bio-medical engineering, human-oriented service robotics, robot assisted rehabilitation, wearable and/or portable orthotics and prosthetics, smart automotive and mechanisms engineering, and so on. The basic key feature of the electrode design has an important initial effect on potential applications in many different industrial fields where the specific contractive property comes to significant importance.

Up to now a few basic experiments have been carried out in order to gain first findings of the electro-mechanical performance and to estimate the future application potential. As soon as one efficient and automated manufacturing process is well established a large number of actuators with varying design parameters can be built for further extensive investigations. Furthermore modeling of the electro-static behavior of the actuator has to be performed to optimize the design- and future manufacturing process.

As a matter of fact the design of the stack actuator is essentially influenced by the electro-mechanical property of the dielectric elastomer. Most stack actuators were made of acrylic material up to now. In order to overcome the resulting limitations and lifetime problems the inherent stress of the pre-strained acrylic film has been reduced by the IPN postprocess. As the benefit of this procedure no additional external supporting structure is necessary, which is an essential requirement for a practical pile-up process. Accordingly the stress reduction of the pre-stretched film represents one of the major milestones to achieve the most efficient and powerful acrylic based DE soft actuator. First tests have been made on silicone based actuators with promising performance results. The aim of this approach was to demonstrate the working principle on non sticky materials. Further extensive investigations have to be provided in order to develop the manufacturing and stacking process of coated thin film in multilayer configuration based on silicon or polyurethane materials.

\section{ACKNOWLEDGEMENT:}

We would like to thank Kurt Gantner from the Swiss Federal Laboratories for Materials Testing and Research (Empa) for their contributions to the present work.

\section{REFERENCES}

1 Ashley S., "Artificial Muscles”, Scientific American, 2003, p 52-59

2 Pelrine R., R. Kornbluh, Q. Pei, S. Stanford, S. Oh and J. Eckerle, "Dielectric Elastomer Artificial Muscle Actuators: Toward Biomimetic Motion" Proc. SPIE in Smart Struct. and Mat.: Electroactive Polymer Actuators and Devices, San Diego (USA), vol. 4695, p 126-37, 2002

3 Bar-Cohen Y., "Electroactive Polymer (EAP) Actuators as Artificial Muscles - Reality, Potential and Challenges" (Ed: Y. Bar-Cohen), SPIE, Bellingham, 2001

4 Kornbluh R., "Dielectric elastomer artificial muscle for actuation, sensing, generation, and intelligent structures", Mat. Techn., 19(4), p 216-24, 2004

5 Pelrine R. E., R. D. Kornbluh and J. P. Joseph, "Electrostriction of polymer dielectrics with compliant electrodes as a means of actuation", Sensors and Actuators A: Physical, 64(1), p 77-85, 1998

6 Y. Bar-Cohen (ed.), Electroactive Polymer (EAP) Actuator as Artificial Muscles: Reality, Potential, and Challenges Second Edition, SPIE Press, Bellingham, Washington, 2004

7 R. Pelrine, R. Kornbluh, and G. Kofod, "High-Strain Actuator Materials Based on Dielectric Elastomer", Advanced Materials 12, 1223-1225, 2000

8 R. Perine, R. Kornbluh, Q. Pei, and J. Joseph, "Hight-Speed Electrically Actuated Elastomers with Strain Greater than $100 \%$ ", Science 287, 836-839, 2000 
9 K. Meijer, M. Rosenthal, and R. J. Full, “ Muscle-Like Actuators? A Comparison between Three Electroactive Polymer”, Proc. SPIE Int. Soc. Opt. Eng. 4329, 7-15, 2001

10 G. Kofod, R. Kornbluh, R. Pelrine, and P. Sommer-Larsen, "Actuation Response of Polyacrylate Dielectric Elastomer”, Proc. SPIE Int. Soc. Opt. Eng. 4329, 141-147, 2001

11 P. Sommer-Larsen, G. Kofod, S. MH, M. Benslimane, and P. Gravesen, "Performance of Dielectric Elastomer Actuators and Materials", Proc. SPIE Int. Soc. Opt. Eng. 4695, 158-166, 2002

12 Pei Q, Pelrine R, Standford S, Kornbluh R and Rosenthal M 2003 Electroelastomer rolls and their application for biomimetic walking robots, Synth. Met. 135-136 129-31

13 Pei Q, Rosenthal M, Stanford S, Prahlad H and Pelrine R 2004 Multiple-degrees-of-freedom electroelastomer roll actuators, Smart Materials and Structures 13(5) N86-N92

14 Q. Pei, M. A. Rosenthal, R. Pelrine, S. Stanford, and R. D. Kornbluh. Multifunctional electroelastomer roll actuators and their application for biomimetic walking robots. In Proc. of SPIE Smart Struct. and Mat.: Electroactive Polymer Actuators and Devices (EAPAD), volume 5051, pages 281-290, San Diego (USA), 2003

15 R. Zhang, P. Lochmatter, A. Kunz, and G. Kovacs., spring roll dielectric elastomer actuators for a portable force feedback glove, SPIE Smart Struct. and Mat.: Electroactive Polymer Actuators and Devices (EAPAD), volume 6168, pages 505-516, San Diego (USA), 2006

16 F. Carpi et al., 'Dielectric Elastomers as Electromechanical Transducers", Elsevier, 2008

17 Kovacs G., Lochmatter P., Wissler M. 2007 Arm Wrestling Robot Driven by Dielectric Elastomer Actuators Smart Materials and Structures 16 No 2 S306-S317

18 F. Carpi and D. De Rossi. Contractile dielectric elastomer actuator with folded shape. In Proc. of SPIE Smart Struct. and Mat.: Electroactive Polymer Actuators and Devices (EAPAD), volume 6168, pages 99-104, San Diego (USA), 2006

19 Jungmann, M. and H. F. Schlaak (2002). Electrostatic Actuators with Elastic Dielectric for Use on Tactile Displays. 8th International Conference on New Actuators, Bremen, Germany

20 Ha S M, Yuan W, Pei Q, Pelrine M and Stanford S 2006 New high-performance electroelastomer based on interpenetrating polymer networks Proc. SPIE in Smart Struct. and Mat.: Electroactive Polymer Actuators and Devices (San Diego, USA) 6168 08-1-08-12

21 G. Kovacs et al., New contractive tension force dielectric EAP actuator, Sensors and Actuators, submitted Feb. 2009 